\title{
Interference and the lossless lossy beam splitter
}

\author{
JOHN JEFFERS \\ Department of Physics and Applied Physics, \\ University of Strathclyde, \\ 107 Rottenrow, \\ Glasgow G4 0NG, UK. \\ email: john@phys.strath.ac.uk
}

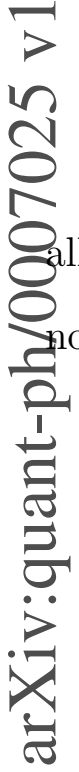




\section{Introduction}

The beam splitter is the main component of many optical interferometers, both classical and quantum [1,2]. Much of its usefulness in quantum optics is derived from the fact that an unentangled input beam can emerge from the device in an entangled state. This state can then either have its quantum correlations confirmed by measurement, or can itself be used in interference experiments. Two photon interference is a higher order quantum interference effect which has received much attention [3 6]. In the classic experiments two identical photons from different input arms falling simultaneously upon a 50/50 beam splitter both exit the beam splitter from the same output arm; whereas if the photons arrive at the beam splitter in the same input they are distributed between the output arms as if they obeyed the laws of classical probability, with each having a transmission and reflection probability of $1 / 2$.

The relations between the input and output single-mode boson operators for the different arms of a lossless beam splitter (Fig. 1) are [7]

$$
\begin{aligned}
& \hat{a}_{\text {out }}=t \hat{a}_{\text {in }}+r \hat{b}_{i n}, \\
& \hat{b}_{\text {out }}=t \hat{b}_{\text {in }}+r \hat{a}_{i n},
\end{aligned}
$$

where $t$ and $r$ are the transmission and reflection coefficients. Throughout this paper discrete-mode theory is used. It is merely an approximation to the more correct continuous-mode formalism, but it is an adequate description here, provided that the reflection and transmission coefficients can be treated as constants at the frequencies of interest. Conservation of the boson commutators and the requirement that independent inputs give independent outputs force the coefficients to satisfy $|t|^{2}+|r|^{2}=1$ and $t= \pm i r$. It is useful to define superposition modes, the symmetric and

antisymmetric combinations of $\hat{a}$ and $\hat{b}$. The annihilation operators for these are (for either inputs or outputs)

$$
\hat{c}=\frac{\hat{a}+\hat{b}}{\sqrt{2}}, \hat{d}=\frac{\hat{b}-\hat{a}}{\sqrt{2}},
$$




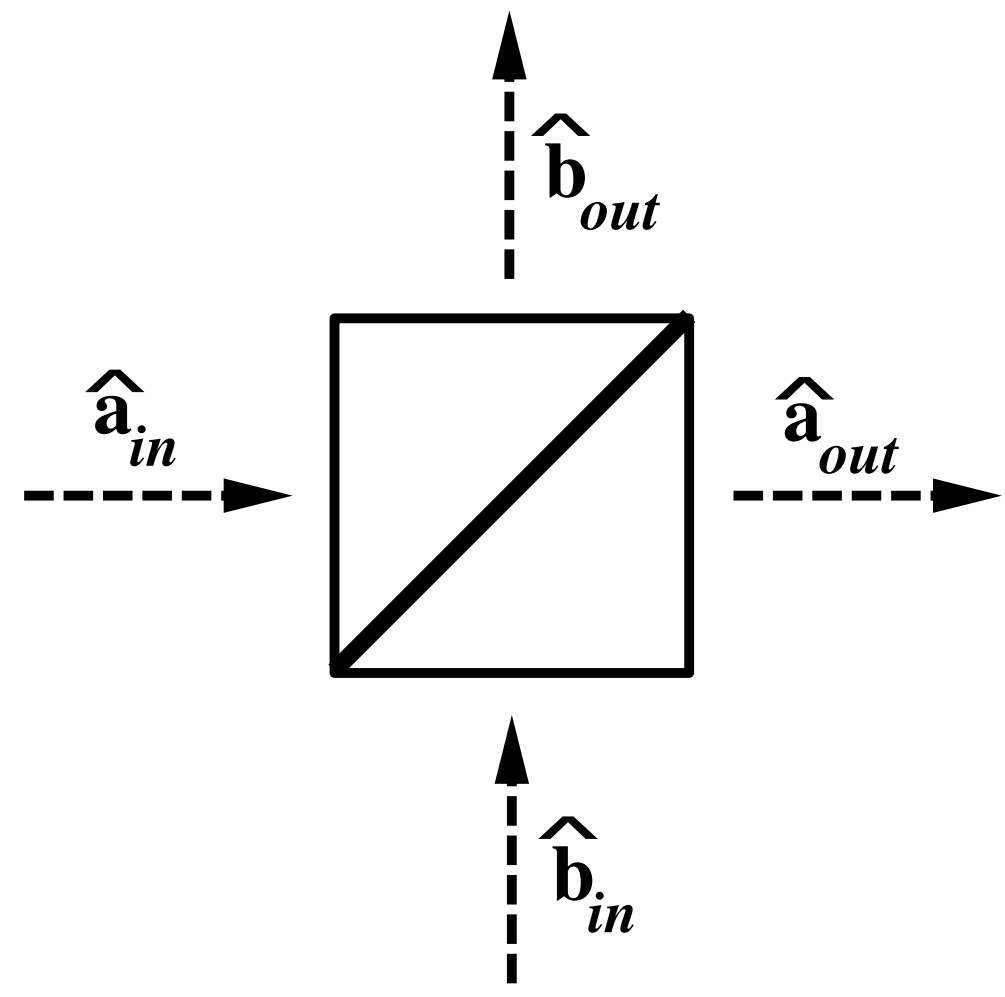

Figure 1: The optical beam splitter. Two input fields are partially reflected, partially transmitted into two output fields. In the lossy case there are further unobservable noise inputs and outputs, which are internal to the beam splitter.

so that the input and output operators are related by

$$
\hat{c}_{\text {out }}=(t+r) \hat{c}_{\text {in }}, \hat{d}_{\text {out }}=(t-r) \hat{d}_{\text {in }} .
$$

It is clear from the above relations that these superposition operators are not mixed by the beam splitter. The action of the device on any input state which is factorisable into a product state of the superposition modes, $\left|\psi_{i n}\right\rangle=\left|\psi_{c}\right\rangle \otimes\left|\psi_{d}\right\rangle$, is simply to produce a phase shift. For example, the photon number state product

$$
\left|\psi_{\text {in }}\right\rangle=\left|n_{c}\right\rangle \otimes\left|m_{d}\right\rangle \Rightarrow\left|\psi_{\text {out }}\right\rangle=\exp [ \pm i \theta(n-m)]\left|n_{c}\right\rangle \otimes\left|m_{d}\right\rangle,
$$

where $\cos \theta=t$ and $|\sin \theta|=r$. For the $50 / 50$ beam splitter two simple examples are the one photon superpositions

$$
\left|1_{+}\right\rangle=\left|1_{c}, 0_{d}\right\rangle=\frac{1}{\sqrt{2}}\left(\left|1_{a}, 0_{b}\right\rangle+\left|0_{a}, 1_{b}\right\rangle\right),
$$




$$
\left|1_{-}\right\rangle=\left|0_{c}, 1_{d}\right\rangle=\frac{1}{\sqrt{2}}\left(\left|1_{a}, 0_{b}\right\rangle-\left|0_{a}, 1_{b}\right\rangle\right) .
$$

It is also possible to construct states for which the beam splitter is transparent with two photons. Consider an input in one of the superpositions

$$
\left|2_{ \pm}\right\rangle=\frac{1}{\sqrt{2}}\left(\left|2_{a}, 0_{b}\right\rangle \pm\left|0_{a}, 2_{b}\right\rangle\right) .
$$

Two photons falling on a beam splitter from the same input arm are usually distributed between the outputs according to the laws of classical probability but this is not the case here. Photons in the positive superposition are constrained to take different output arms. This is an illustration of the reversibility of light beams in optics, as it is merely the standard two photon interference phenomenon with the inputs and outputs reversed. The input state in this case is not factorisable in terms of states of the $c$ and $d$ modes. The negative superposition, on the other hand, is a two photon example of a state which passes through the beam splitter unchanged. This state can also be written

$$
\left.\left|2_{-}\right\rangle=\frac{1}{2}\left(\hat{a}^{\dagger 2}-\hat{b}^{\dagger 2}\right)\left|0_{a}, 0_{b}>=-\hat{c}^{\dagger} \hat{d}^{\dagger}\right| 0_{c}, 0_{d}\right\rangle .
$$

The reason that the state is passed is now clear. It is factorisable into a product state of the superposition modes.

Much of the theory of the beam splitter has been done assuming that it is a lossless component [7 10], for which absorption is simply an experimental problem that hinders the observation of interesting effects. Absorption is unavoidable at some frequencies, however, as optical devices must be compatible with the Kramers-Kronig relations. In recent years loss has come to be seen as a quantum property in its own right, with its own effect on correlations [11]. There has been some work on loss in fibre couplers [12] but it is only recently that a quantum theory of the absorbing beam splitter was proposed [13]. All of the features of the lossless device are retained but there are additional quantum effects such as nonlinear absorption which are only possible when loss is present.

In this paper a discrete-mode version of the lossy beam splitter theory is used to show several interesting effects. Firstly, the beam splitter may be rendered transparent or opaque, depending on 
the phase relation of the transmission and reflection coefficients and the state of the input light; and secondly, the nonlinear absorption effect discussed in reference [13] is shown to occur with other two photon input states.

\section{The lossy beam splitter}

In this section the theory of the lossy beam splitter [13] will be outlined in discrete-mode form. The device relates two inputs to two outputs as before (Fig. 1), but the presence of absorption forces changes to the theory. The beam splitter input and output operators are related by

$$
\begin{aligned}
& \hat{a}_{\text {out }}=t \hat{a}_{\text {in }}+r \hat{b}_{i n}+\hat{f}_{a}, \\
& \hat{b}_{\text {out }}=t \hat{b}_{\text {in }}+r \hat{a}_{i n}+\hat{f}_{b},
\end{aligned}
$$

where in this case $|t|^{2}+|r|^{2}<1$. The noise operators, $\hat{f}_{a}$ and $\hat{f}_{b}$, are necessary to preserve the commutators of the observable outputs. We will assume that the noise modes are unexcited, and can thus make no contribution to output photocounts. Furthermore, the reflection and transmission coeffecients are not constrained by a phase relation similar to the one for the lossless beam splitter (that is, $t \neq i r$ in general). The requirement that the beam splitter is lossy for coherent inputs does, however, give another relation which the reflection and transmission coefficients must satisfy,

$$
|t \pm r|<1
$$

The superposition operators for the lossy beam splitter are simply the sum and difference operators as before. The output operators are appended by noise operators,

$$
\begin{aligned}
& \hat{c}_{\text {out }}=(t+r) \hat{c}_{\text {in }}+\hat{f}_{c}, \\
& \hat{d}_{\text {out }}=(t-r) \hat{d}_{\text {in }}+\hat{f}_{d},
\end{aligned}
$$

where $\hat{f}_{c}$ and $\hat{f}_{d}$ are respectively the sum of and difference between the noise operators $\hat{f}_{a}$ and $\hat{f}_{b}$. The theory outlined above is sufficient for this paper. A fuller account of the general theory in a continuous mode formalism is given in reference [13]. 


\section{The lossless lossy beam splitter}

Consider a lossy beam splitter with transmission and reflection coefficients which are equal or opposite, $t= \pm r$. Such a phase relation would not be allowed for the lossless beam splitter, but the extra freedom given by the noise operators associated with absorption means that it is an allowed choice. There is, however, one restriction imposed by equation (9) which limits the moduli of $t$ and $r$ to be less than or equal to $1 / 2$. The result of this is at best a $25 / 25$ beam splitter with $50 \%$ loss. The effects described also occur with reduced visibility for beam splitters with greater absorption than $50 \%$ but it is this ideal case which is assumed henceforth. It is clear that for this choice of phase relation one of the superposition modes is passed completely and the other is fully absorbed. The $\left|1_{ \pm}\right\rangle$states are the one-photon excitations of these superposition operators, and Table 1 summarises the effect of the beam splitter on these inputs.

Table 1

\begin{tabular}{|l|l|l|}
\hline input & beam splitter & output \\
\hline \hline \multirow{2}{*}{$\left|1_{+}\right\rangle$} & $t=r$ & $\left|1_{+}\right\rangle$ \\
\cline { 2 - 3 } & $t=-r$ & $|0\rangle$ \\
\hline \multirow{2}{*}{$\left|1_{-}\right\rangle$} & $t=r$ & $|0\rangle$ \\
\cline { 2 - 3 } & $t=-r$ & $\left|1_{-}\right\rangle$ \\
\hline
\end{tabular}

Table 1 . One photon superposition input states and output states for the $25 / 25$ lossy beam splitter.

Clearly the photons are passed or absorbed with either unit or zero probability. They are not subject to the randomness normally associated with an absorbing medium that plagues other input states. Although the table illustrates the results with one photon states, the effect is not specifically quantum in nature. The beam splitter will be rendered either transparent or opaque to any excitation of the superposition modes, even a classical excitation such as the coherent state. The device exhibits the remarkable property that even though half of the input light is normally lost, the full intensity can be obtained at the outputs if the input is in a particular mode. Furthermore, it is relatively easy to put input light into a beam splitter in one of the superposition modes. A Mach-Zehnder interferometer with the second beam splitter as the lossy one could be set up to do 


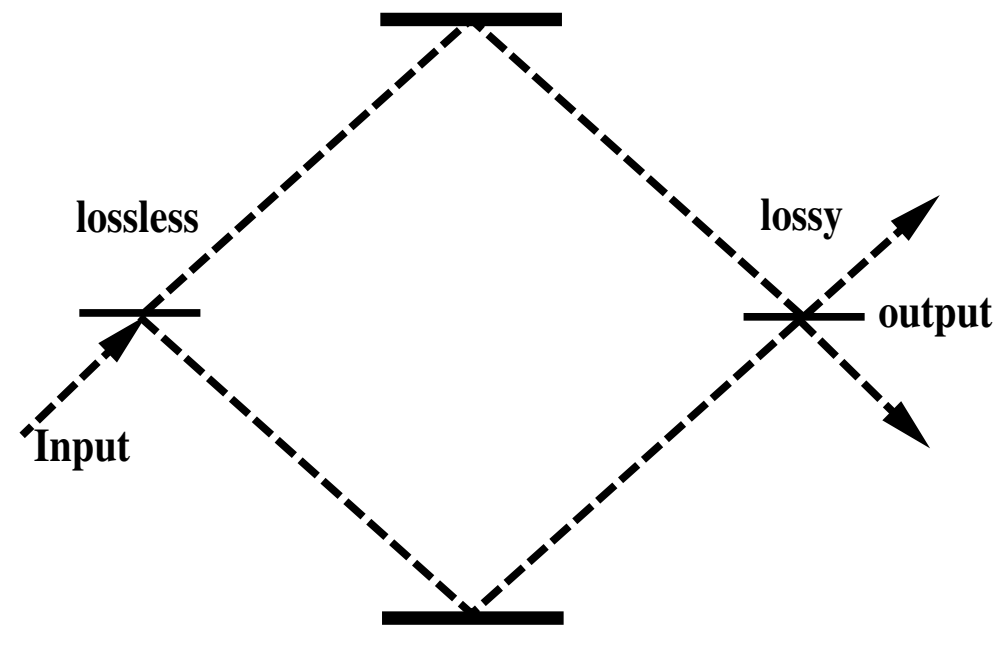

Figure 2: Mach-Zehnder set up to obtain an input into the lossy beam splitter in one of the superposition modes. The first beam splitter is a standard lossless 50/50 device, the second is lossy, $25 / 25$.

this for narrow band input light (Fig. 2).

\section{Two photon interference and nonlinear absorption}

The previous section described how a beam splitter can be made transparent (or opaque) for classical or quantum excitations of the superposition modes. In this section the effects of the absorbing beam splitter on purely quantum input states are described. The standard two photon interference experiment, in which a pair of photons incident on a beam splitter from different input arms produce no coincidences at the outputs, survives with reduced visibility in the lossy case provided that the same phase relation $t= \pm i r$ is upheld. The same is true of the extensions to two photon interference described in the Introduction. If, however, the beam splitter has equal or opposite $t$ and $r$, nonlinear absorption can take place. It has previously been pointed out that for an input state with one photon in each input arm it is not possible to obtain a one photon output [13. Either both photons pass through or both are absorbed. There are similar nonlinear phenomena which occur when the input is in one of the two-photon superposition states (6). These are summarised in Table 2. 
Table 2

\begin{tabular}{|l|l|}
\hline input & output $(t= \pm r)$ \\
\hline \hline$\left|1_{a}, 1_{b}\right\rangle$ & mixture of $\left|0_{a}, 0_{b}\right\rangle$ and $\frac{1}{\sqrt{2}}\left(\left|1_{a}, 1_{b}\right\rangle \pm\left|2_{+}\right\rangle\right)$ \\
\hline$\left|2_{+}\right\rangle$ & mixture of $\left|0_{a}, 0_{b}\right\rangle$ and $\frac{ \pm 1}{\sqrt{2}}\left(\left|1_{a}, 1_{b}\right\rangle \pm\left|2_{+}\right\rangle\right)$ \\
\hline$\left|2_{-}\right\rangle$ & $\mp\left|1_{ \pm}\right\rangle$ \\
\hline
\end{tabular}

Table 2. Two photon input states and output states for the 25/25 lossy beam splitter.

Clearly nonlinear absorption takes place in each case. For the input states specified in the first two lines of Table 2 the outputs cannot consist of only one photon, whereas the input state in the third line produces an output of one photon with certainty. In fact nonlinear interference occurs for two photon input superpositions with any relative phase angle (not merely 0 or $\pi$ as in the Table 2). There is a simple explanantion for the nonlinear interference in terms of the superposition operators. The input state from the first line of the Table 2 can be written

$$
\left|1_{a}, 1_{b}\right\rangle=\hat{a}_{i n}^{\dagger} \hat{b}_{i n}^{\dagger}\left|0_{a}, 0_{b}\right\rangle=\frac{1}{2}\left(\hat{c}_{i n}^{\dagger 2}-\hat{d}_{i n}^{\dagger 2}\right)\left|0_{c}, 0_{d}\right\rangle
$$

when expressed in terms of the superposition operators for the inputs. One of $\hat{c}$ or $\hat{d}$ is completely absorbed by the beam splitter, so that the part of the output which passes is either $\hat{c}_{\text {out }}^{\dagger 2} / 2$ or $-\hat{d}_{\text {out }}^{\dagger 2} / 2$ acting on the vacuum. The output state, found by re-expressing these operators in terms of the standard output operators, is as given in Table 2. Similarly the input state in the second line of

Table 2, $\left|2_{+}\right\rangle$, can be written $\frac{1}{2}\left(\hat{c}_{i n}^{\dagger 2}+\hat{d}_{i n}^{\dagger 2}\right)\left|0_{c}, 0_{d}\right\rangle$, so the explanation, and the output state are the same (except for a simple phase change in one case). The odd state out is the input in the third line of the Table 2, which from equation (7) is $-\left|1_{c}, 1_{d}\right\rangle$. There is therefore one photon in each of the input superposition modes, one of which is absorbed and the other passes. Hence the output must contain one photon in the passed superposition mode.

\section{Conclusions}

In this paper it has been shown that the amount of absorption produced by a lossy beam splitter can be reduced if the transmission and reflection coefficients have an appropriate phase relation and the input light is directed into particular modes. For a 50\% lossy beam splitter, which ordinarily 
would absorb half of the light, it is possible to obtain all of the input light in the output, thus rendering the device transparent.

The modes which are affected are the superposition modes, the sum and difference of the two inputs. One photon excitations of these modes are quantum states which are useful in quantum information [14]. The lossy beam splitter described here could therefore be used as a filter, absorbing merely that part of the input which is not in the required superposition.

A further consequence of the different amount of loss in each superposition is the possibility of nonlinear absorption from a linear device. For some two photon inputs the chance of a one photon output can be eliminated. On the other hand for the two photon input which is factorisable in terms of superposition input states, a single photon output is obtained with certainty. It is clear from the above that, in both the classical and quantum cases, interference is not simply washed out by absorption. Loss can enhance interference, and it even has effects of its own which are not present in lossless environments.

\section{Acknowledgments}

This research was supported by the Engineering and Physical Sciences Research Council. I would like to thank Steve Barnett for useful comments. 


\section{References}

[1] see, for example, Mandel, L. and Wolf, E., 1995, Optical Coherence and Quantum Optics, (Cambridge: Cambridge University Press).

[2] Hanbury-Brown, R. and Twiss, R.Q., 1956, Nature 177, 27 .

[3] Hong, C. K., Ou, Z. Y., and Mandel, L., 1987, Phys. Rev. Lett. 59, 2044.

[4] Rarity, J. and Tapster, P. R., 1990, in Coherence and Quantum Optics VI, Proceedings of the Sixth Rochester Conference on Coherence and Quantum Optics, edited by J.H. Eberly, L. Mandel and E. Wolf (New York: Plenum); 1988, in Photon Localisation, Detection, Amplification and Antibunching, edited by E.R. Pike and H. Walther (Bristol: Hilger).

[5] Steinberg, A.M., Kwiat, P.G. and Chiao, R.Y., 1992, Phys. Rev. Lett. 68, 2421.

[6] Loudon, R., 1990, in Coherence and Quantum Optics VI, Proceedings of the Sixth Rochester Conference on Coherence and Quantum Optics, edited by J.H. Eberly, L. Mandel and E. Wolf (New York: Plenum); 1998, Phys. Rev. A, 58, 4904.

[7] Prasad, S., Scully, M.O. and Martienssen, W., 1987, Opt. Commun. 62, 139.

[8] Ou, Z.Y., Hong, C.K. and Mandel, L., 1987, Opt. Commun. 63, 118.

[9] Fearn, H. and Loudon, R., 1987, Opt. Commun. 64, 485.

[10] Campos, R.A., Saleh, B.E.A. and Teich, M.C., 1989, Phys. Rev. A 40, 1371.

[11] Jeffers, J. and Barnett, S. M., 1993, Phys. Rev. A, 47, 3291; 1994, J. Mod. Opt., 41, 1121.

[12] Lai, W.K., Bužek, V. and Knight, P.L., 1991, Phys. Rev. A 43, 6323.

[13] Barnett, S. M., Jeffers, J., Gatti, A. and Loudon, R., 1998, Phys. Rev. A, 57, 2134.

[14] Bruss, D., Ekert, A., Huelga, S.F., Pan, J.-W. and Zeilinger, A., 1997, Phil. Trans. Roy. Soc. A, 355, 2259. 